\title{
Numerical Analysis of Droplet Deposition in Inkjet Printed Electronics Assembly
}

\author{
T. Tilford*, S. Stoyanov, G. Tourloukis, C. Bailey \\ The University of Greenwich, Park Row, London, UK \\ T.Tilford@gre.ac.uk
}

\begin{abstract}
In this paper, a computational approach for the analysis of microscale droplet impact dynamics is presented. The approach is intended to support a condition based monitoring system to enhance quality and reliability of inkjet printed electronics components. The Smoothed Particle Hydrodynamics (SPH) approach of Lucy and Gingold and Monaghan has been used as the basis for the model, with the $\delta$-SPH terms of Marrone et al used to improve handling of the dynamic impact events and the gradient correction terms of Belytschko used to improve the accuracy of interface dynamics.
\end{abstract}

Model validation has been performed through comparison against a macroscale dam break problem and through a microscale analysis designed to determine accurate surface tension-pressure behaviour based on the Young-Laplace relation. The model is used to assess impact of a single drop on a uniform surface and the three dimensional formation of multi-drop layers

\section{Introduction}

Piezoelectric drop-on-demand inkjet printing systems are increasingly becoming tools for additive manufacturing of complex high value products. These systems can be used to form truly three dimensional, multi material objects with very high dimensional accuracy. The development of conductive pastes that can be dispensed using inkjet printers has lead to the approach being utilised for development of microelectronics components. A number of academic research teams and commercial companies have used inkjet systems to form microelectronics systems (e.g. Kawahara et al, [1] as illustrated in Figure 1). The complexity of the approach is increasing rapidly, with systems such as the NextFactory concept [2] incorporating multiple material print capability with ultra-precise electronics assembly and inspection capabilities. The ability to form complete customised components using a single piece of equipment in a relatively short period of time is a highly attractive prospect for electronics manufacturers.

As is the norm for the electronics sector, new manufacturing approaches need to be considered in terms of the long term reliability of the final product. In addition to commonplace reliability qualification approaches such as JEDEC tests, there is an increasing drive to assess component quality during the manufacturing process.
Condition based monitoring approaches measure key parameters associated with component quality during manufacture and continually optimise process parameters in real time to increase final quality and reliability of formed components [3]. Such condition based monitoring systems need to be trained as to how variation of process parameters influences product quality. A numerical model, capable of detailed analysis of the process, can be used to underpin such an approach.

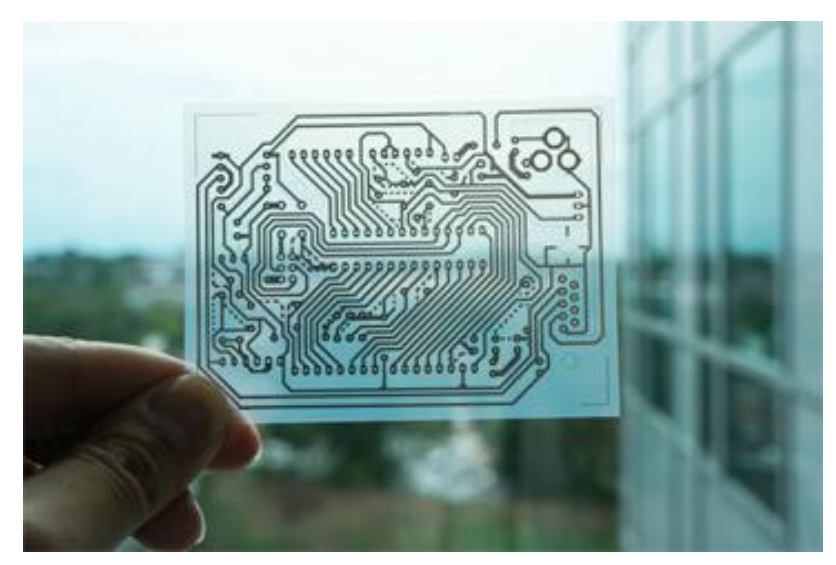

Figure 1: Printed microcontroller (Georgia Tech)

The primary requirement of the numerical model for inkjet deposition is to capture the complex physics involved when and inkjet droplet impacts a printed surface. There are a number of significant challenges in such an analysis. The primary challenge is that analysis of droplet impact upon an idealised flat surface is insufficient. Only the first layer of an inkjet printed structure will be deposited on the flat baseplate. The following layers will be deposited onto a layer of partially cured polymer droplets which form and uneven surface and will deform on impact. The material is not a simple Newtonian fluid such as water but a complex multicomponent polymer which exhibits shear dependent viscous behaviour - a complex non-Newtonian material. Additionally, the impact is very severe with a droplet of diameter in the order of 40 microns impacting at approx. 5 metres per second.

Traditional computational fluid dynamics (CFD) approaches such as the Finite Volume Method [4] would be readily capable of modelling the impact dynamics of a small number of droplets. However, in order to consider prediction of the development of defects over a number of layers it is necessary to take advantage of a more efficient 
approach such as GPU enabled SPH. This approach has a number is advantages over traditional methods in that interfaces are explicitly captured rather than needing to be approximated but, more critically, incorporates a finite support distance enabling the problem domain to be subdivided into a large number of overlapping subdomains which can be assessed on a single core of a graphical processor unit .

\section{Numerical Approach}

The Smoothed Particle Hydrodynamics (SPH) approach was developed by Lucy [7] and by Gingold and Monaghan in 1977 [8]. It is a versatile discrete particle method for solution of a number of differing physical phenomena. It is a computationally highly effective method for solution of complex fluid flows, particularly in cases with interfaces and large deformations. The SPH approach considers the fluid as a collection of particles, each associated to a number of physical properties such as position, velocity, mass, density, etc. At the heart of the SPH approach is a means of evaluating spatial derivatives through integral interpolants which use kernels to approximate a delta function. The integral interpolant of any quantity function $\mathrm{A}(\mathrm{r})$ is defined by:

$$
A(r)=\int_{\Omega} A\left(r^{\prime}\right) W\left(r-r^{\prime}, h\right) d x
$$

This relates the value of parameter A, a scalar variable such as pressure, at location $r$, through integration of the value of A over surrounding space $\Omega$ with a smoothing kernel W. This smoothing kernel essentially acts as a weighting factor which, critically, enables the variation of A at distances greater than a defined value to be ignored. This finite support radius enables the physical domain to be subdivided into a number of overlapping subdomains which greatly enhances the computational efficiency of the approach. In the standard SPH formulation, this can be written as:

$$
A_{i}(r)=\sum_{j} A_{j} \frac{m}{\rho} W(r i-r j, h)
$$

In which the value of $\mathrm{A}$ of particle $\mathrm{i}$ is evaluated by summing the values of $A$ at all particles within the support radius as a function of their mass, m, density, $\rho$ and kernel, W. This can be extended to spatial derivatives through the following functions:

$$
\begin{aligned}
\nabla A_{i}(r) & =\sum_{j} A_{j} \frac{m}{\rho} \nabla W(r i-r j, h) \\
\nabla^{2} A_{i}(r) & =\sum_{j} A_{j} \frac{m}{\rho} \nabla^{2} W(r i-r j, h)
\end{aligned}
$$

A number of different kernels have been proposed in SPH literature, each with differing behaviour benefits and drawbacks, The cubic spline kernel has been adopted for this analysis as it is the most widely used and understood. The Cubic spline is given by the following function, with normalisation factors, $\sigma$, of $1 / \mathrm{h}, 10 /(7 \pi \mathrm{h} 2)$, and $1 /(\pi \mathrm{h} 3)$ in one, two and three dimensions. Plots of the normalised kernel and kernel derivative are presented in figure 2 while graphical representations of the kernel value and support radius are presented in Figure 3.

$$
W(r, h)=\sigma\left\{\begin{array}{lc}
1-\frac{3}{2} q^{2}+\frac{3}{4} q^{3} & 0 \leq q \leq 1 \\
\frac{1}{2}(2-q)^{3} & 1 \leq q \leq 2 \\
0 & q>2
\end{array}\right.
$$

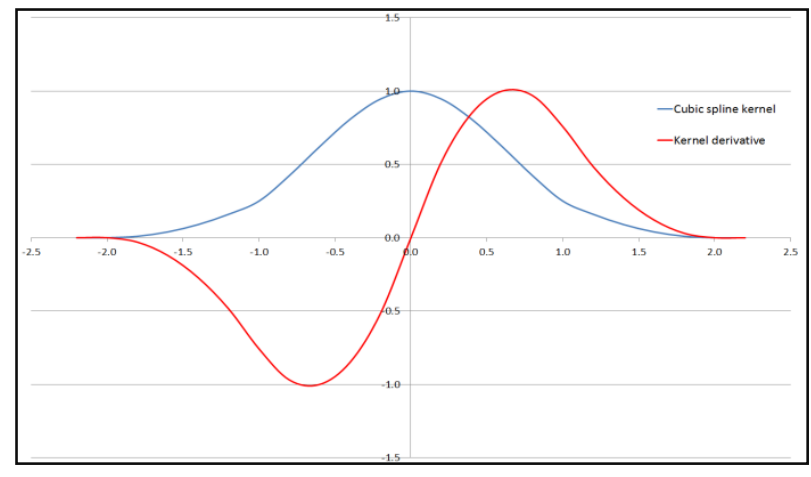

Figure 2: Cubic spline kernel

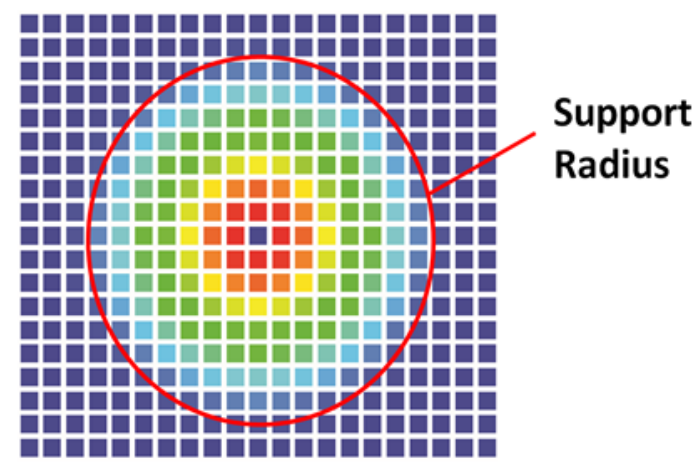

Figure 3: Kernel value and support radius

This limited support radius enables the solution domain to be subdivided into cell each with dimension equal to the support radius. When each cell is linked with the 26 surrounding cells to form a sub-region, the domain is separated into a number of overlapping subdomains in that a particle inside the subregion will only have a valid interaction with particles in the same region as particles in other regions will be more than the support radius away. This is a key advantage of the SPH approach in that the computational cost of solving a number of small problems 
is significantly lower than solving one very large problem. Additionally, the numerical processing can be performed on a graphical processing unit (GPU) which comprises a relatively large number of relatively small cores which is ideally suited to such problems. The GTX Titan black GPU system used for this work has 2880 individual cores with a peak performance of approx. 5.1 TFlops. The SPH subdomains are distributed over the GPU cores as illustrated in figure 4.

Within each subdomain it is necessary to determine the movement of each particle as a function of the acceleration due to interaction forces from surrounding particles. The fluid flow forces are governed by the Navier Strokes Equations, which can be written as:

$$
\frac{\delta}{\delta t}(\rho u)+(\rho u \cdot \nabla)=-\nabla p+\mu \nabla^{2} u+g
$$

In the SPH approach these can be reformulated as a smoothed interaction force between each pair of particles. The acceleration of a particle can therefore be derived through summation of these forces over all particles within the support radius. The total acceleration force can be written as:

$$
\begin{aligned}
\frac{\delta \rho_{i}}{\delta t}= & -\rho_{i} \sum_{j}\left(u_{j}-u_{i}\right) \cdot \nabla W\left(r_{i}-r_{j}, h\right) \frac{m_{i}}{\rho_{i}} \\
\frac{\delta}{\delta t}\left(\rho u_{i}\right)= & -\sum_{j} m_{j}\left[\left(\frac{p_{i}}{\rho_{i}^{2}}+\frac{p_{j}}{\rho_{j}^{2}}\right)\right. \\
& \left.-\frac{\varepsilon}{\rho_{i} \rho_{j}} \frac{4 \mu_{i} \mu_{j}}{\left(\mu_{i}+\mu_{j}\right)} \frac{u_{i j} \cdot r_{i j}}{r_{i j}^{2}+\eta^{2}}\right] \nabla W\left(r_{i}-r_{j}, h\right)
\end{aligned}
$$

In addition to the standard SPH formulation, a number of additional functions needed to be implemented in order to address specific challenges of the inkjet droplet impact problem. The first of these is to implement the dissipative SPH framework of Marrone et al [7] in order to better deal with the violent impact events. This framework involves modification of the interaction forces to incorporate additional stabilisation terms such that:

$$
\begin{aligned}
\frac{\delta \rho_{i}}{\delta t}= & -\rho_{i} \sum_{i}\left(u_{j}-u_{i}\right) \cdot \nabla W\left(r_{i}-r_{j}, h\right) \frac{m_{j}}{\rho_{j}} \\
& +\delta h c_{0} \sum_{j} \psi_{i j} \cdot \nabla W\left(r_{i}-r_{j}, h\right) \frac{m_{j}}{\rho_{j}} \\
\frac{\delta}{\delta t}\left(\rho u_{i}\right)= & -\sum_{j} m_{j}\left[\left(\frac{p_{i}}{\rho_{i}^{2}}+\frac{p_{j}}{\rho_{j}^{2}}\right)\right. \\
& \left.-\frac{\varepsilon}{\rho_{i} \rho_{j}} \frac{4 \mu_{i} \mu_{j}}{\left(\mu_{i}+\mu_{j}\right)} \frac{u_{i j} \cdot r_{i j}}{r_{i j}^{2}+\eta^{2}}\right] \nabla W\left(r_{i}-r_{j}, h\right) \\
& +\alpha h c_{0} \rho_{0} \sum_{i} \pi_{i j} \nabla W\left(r_{i}-r_{j}, h\right) \frac{m_{j}}{\rho_{j}}
\end{aligned}
$$

where:

$$
\psi_{i j}=2\left(\rho_{j}-\rho_{i}\right) \frac{r_{j i}}{\left|r_{i j}\right|^{2}}-\left[\left(\nabla \rho_{i}\right)+\left(\nabla \rho_{j}\right)\right]
$$

$$
\pi_{i j}=\frac{\left(u_{j}-u_{i}\right) \cdot r_{j i}}{\left|r_{i j}\right|^{2}}
$$

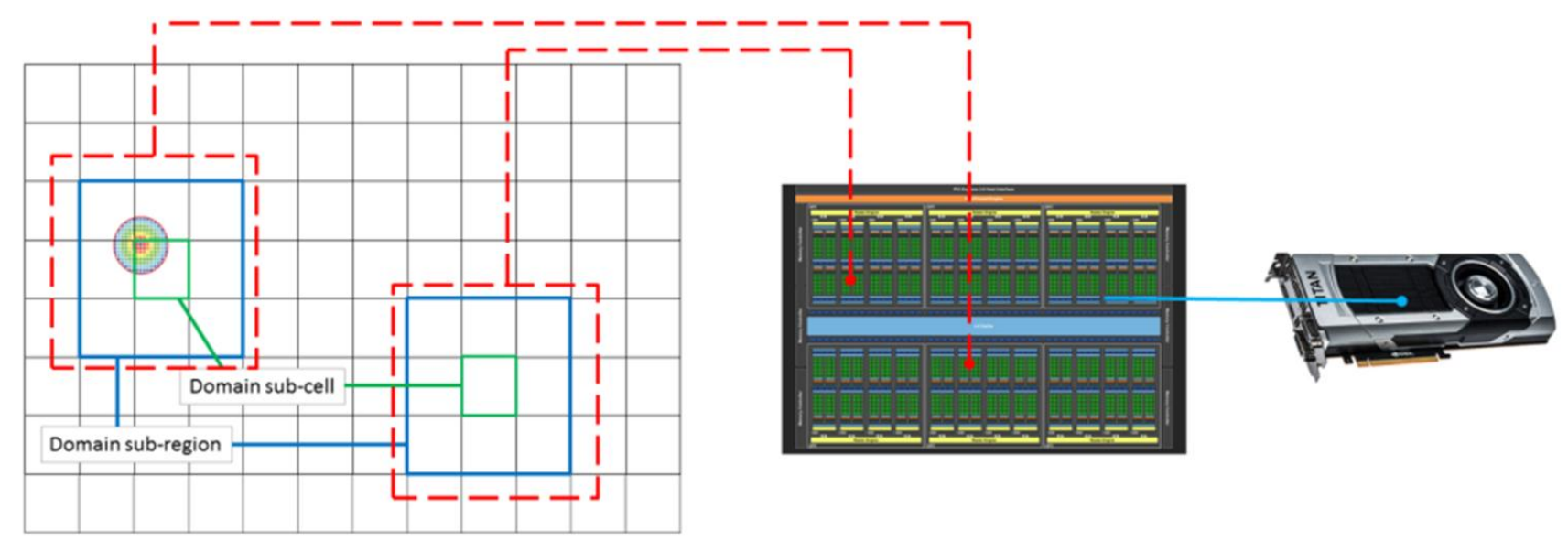

Figure 4: Stream computing domain decomposition 
The XSPH correction of Monaghan [8] has been implemented to stabilise the analysis, which modifies the particle velocity based on the velocity of the surrounding particles in a manner given by:

$$
\frac{\delta r_{i}}{\delta t}=u_{i}+\varepsilon \sum_{j} \frac{m_{b j}}{\bar{\rho}_{i j}} u_{j i} W\left(r_{i}-r_{j}, h\right)
$$

Furthermore, the kernel gradient correction approach of Belytschko [9] is implemented to correct the evaluation of the kernel and gradient values at interfaces. In these regions the support radius covers a region of liquid, represented by particles, and a region of air which, in this implementation, is represented by an absence of particles. The approach of Belytschko requires a $4 \times 4$ matrix to be inverted in order to determine the correction factors however this increases the accuracy of the analysis in the critical impact phase of the process. Time integration has been handled through use of a velocity Verlet scheme [10] while material cure behaviour has been handled through a viscosity modification term. A more detailed analysis of the cure kinetics and the non-Newtonian rheometry of the jetted fluids are required to improve the accuracy of the model.

$$
\begin{gathered}
\boldsymbol{A}=\sum_{j} W_{i}^{S}\left[\begin{array}{cccc}
1 & \delta x & \delta y & \delta z \\
\delta x & \delta x \delta x & \delta y \delta x & \delta z \delta x \\
\delta y & \delta x \delta y & \delta y \delta y & \delta z \delta y \\
\delta z & \delta x \delta z & \delta y \delta z & \delta z \delta z
\end{array}\right] \\
\boldsymbol{A} \boldsymbol{\alpha}=\boldsymbol{I} \\
\mathrm{W}_{i}^{s}=\frac{W_{i}}{\sum_{j}\left(\frac{m_{j}}{\rho_{j}} W_{j}\right)} \\
\nabla W_{x}=\alpha_{21}+\alpha_{22} \delta x+\alpha_{23} \delta y+\alpha_{24} \delta z \\
\nabla W_{y}=\alpha_{31}+\alpha_{32} \delta x+\alpha_{33} \delta y+\alpha_{34} \delta z \\
\nabla W_{z}=\alpha_{41}+\alpha_{42} \delta x+\alpha_{43} \delta y+\alpha_{44} \delta z
\end{gathered}
$$

\section{Model Validation}

Ideally, experimental analysis of the microscale flow phenomena occurring during droplet impact would be used to validate the numerical model. However, no viable data is available at this time. In lieu of this, a series of validation tests have been performed in order to support the validity of the model. It should be noted that development of the model and assessment of key material property parameters is an ongoing process.

Macroscale flow validation has been carried out through comparison with a series of experiments by Buchner [11] considering a dambreak problem. In these experiments, a rectangular water tank is divided into two sections by a movable wall. The wall can be very rapidly withdrawn, resulting in a starting condition comprising an unsupported cuboid of water which will flow into empty space prior to impacting against the far wall. The test case was chosen due to the requirements to accurately capture the impact dynamics. The numerical model provided an accurate prediction of the flow dynamics, with results closely matching those of Buchner and also the numerical analysis of Marrone et al [7]. Figure 5 shows a series of velocity plots showing the initial condition and five subsequent flow states.
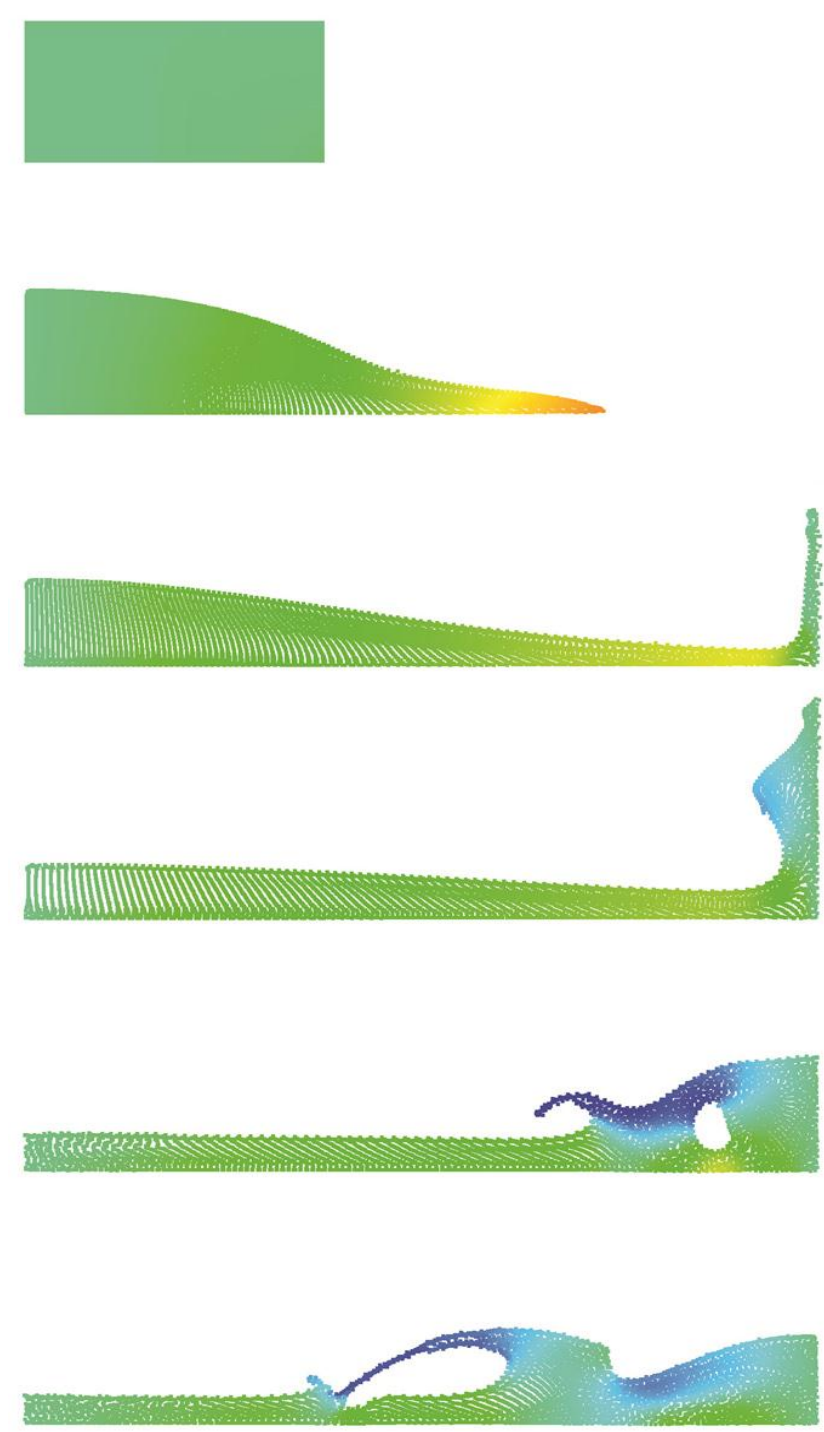

Figure 5: Dambreak flow development 


\section{Young Laplace Surface Tension test}

The influence of surface energy effects become increasingly important as analysis lengthscales decrease. When assessing microscale phenomena such as inkjet droplet dynamics, these surface tension forces typically dominate momentum forces. In order to validate the implantation of surface tension forces within the model an analysis of the development of a cuboid droplet at zero pressure to a spherical droplet at an elevated pressure has been performed. The change in shape and pressure is in response to the surface energy effects, with the final radius and pressure given by the Young-Laplace equation [12-14]. The equation correlates the pressure difference to the mean curvature of the droplet as given by:

$$
\Delta P=\gamma\left(\frac{1}{R_{1}}+\frac{1}{R_{2}}\right)
$$

In which $\gamma$ is the interface surface energy and R1 and R2 are the principle curvatures. Figure 6 shows the evolution of the droplet shape, while Figure 7 shows the pressure variation predicted by the model and the analytical equilibrium state solution of $3.08 \mathrm{fg} \mathrm{um}^{-1} \mathrm{us}^{-2}$.
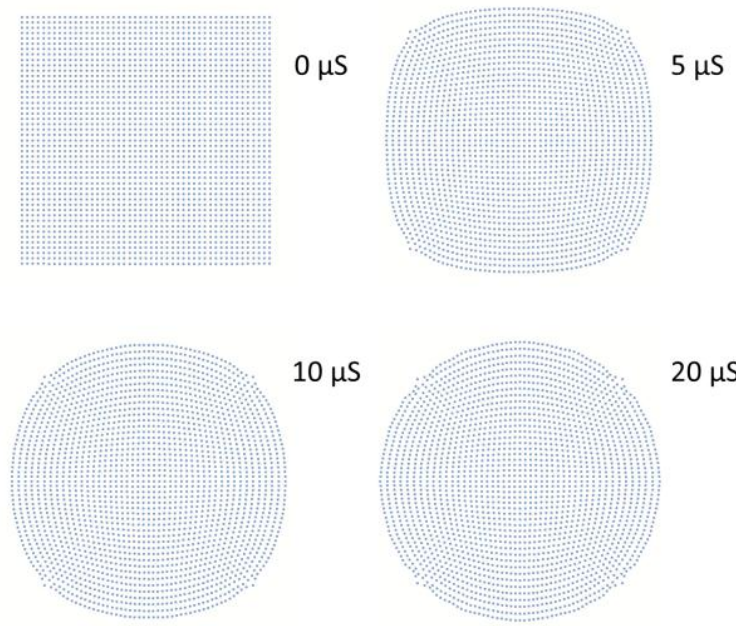

$20 \mu \mathrm{S}$

Figure 6: Young-Laplace shape evolution

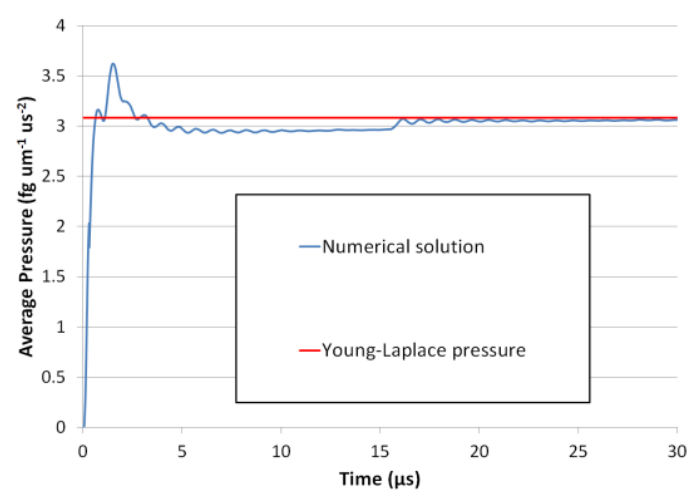

Figure 7: Young-Laplace pressure evolution

\section{Single Droplet Impact Analysis}

Analysis of a single droplet of uncured polymer has been performed using the model. The analysis has considered an initial state of a perfectly spherical droplet of diameter $23 \mu \mathrm{M}$ travelling toward a flat plane at $5 \mathrm{Ms}^{-1}$. The fluid is considered to have constant viscosity of 0.015 $\mathrm{Pa} . \mathrm{S}$ and density $1000.0 \mathrm{KgM}^{-3}$. Surface energy values for the fluid-air interface and fluid surface interface were taken as $72 \mathrm{mJM}^{-2}$. Polymer materials typically exhibit non-Newtonian behavior and the surface energy behavior is more complex than considered in the model and as such the accuracy of the analysis will be limited until the model is extended to capture these phenomena.

The development of the droplet shape during the impact, as predicted by the numerical model, is illustrated in Figure 8. The six images show the droplet at 1, 10, 20, 86,200 and $400 \mu \mathrm{s}$ after impact. The high impact speed causes relatively localized deformation in the immediate post impact phase before the kinetic energy is transferred into transverse momentum and significant viscous energy dissipation. The point at which the droplet has greatest transverse radius occurs at $86 \mu \mathrm{s}$, where momentum forces have been balanced by the surface tension forces resulting in zero velocity at the outermost extents of the droplet. Beyond this time, the surface tension forces draw the droplet back into a more spherical shape as shown in in the 200 and $400 \mu$ s plots.
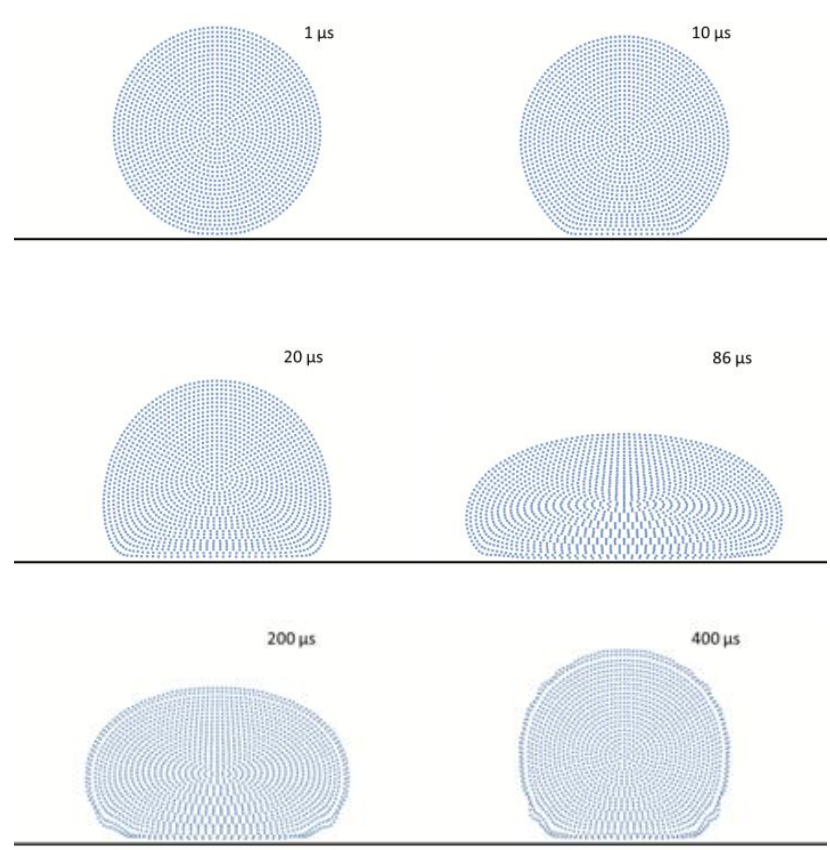

Figure 8: Single droplet impact sequence 


\section{D Printing Analysis}

In order to demonstrate formation of 3D structures from inkjet droplet deposition using the implemented modelling framework an analysis of a simple test problem has been performed. In this case the printing of four layers of the isolating polymer material has been simulated. Each layer is formed of an array of 16 droplets, with each layer being fully cured after deposition. This should be considered as a simplified analysis as the capabilities of the solver extend well beyond the 4 layers, 64 droplets and 1 material with a potential capability of analysing many thousands of droplets formed from multiple materials.
The deposition sequence is illustrated in figure 9. In image 1 of the figure, we can see the four by four droplet array being deposited on the baseplate. On impact the droplets spread and partially coalesce. After deposition of the layer the droplets are cured. This is not readily apparent in image 2 as only the viscosity of the material is altered in this analysis. Image 3 shows the deposition of the second layer, which is subsequently cured (image 5). This process is repeated for layers 3 and 4 with the resultant final shape shown in figure 9.

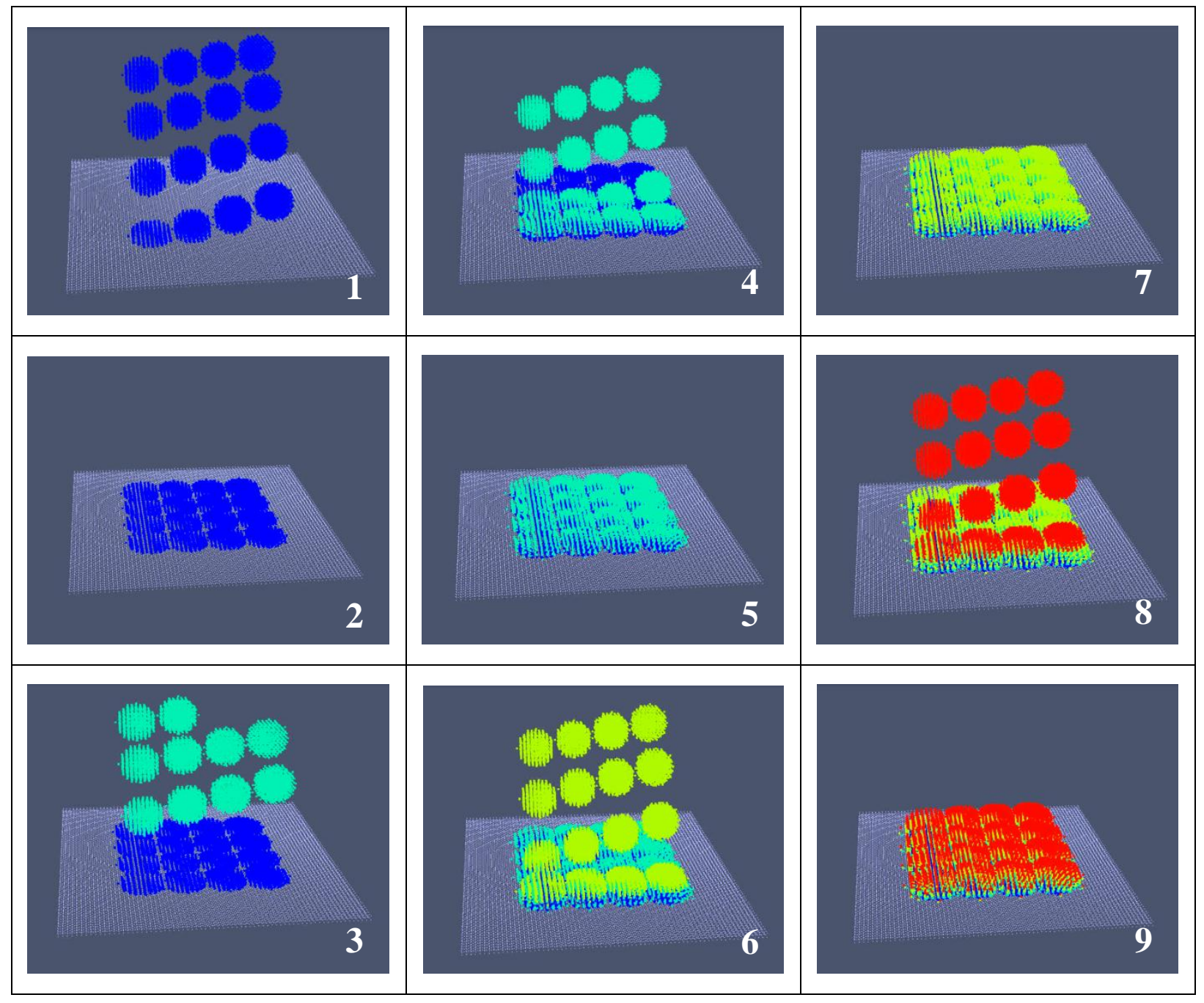

Figure 9: Demonstration droplet deposition sequence 


\section{Conclusions}

A new, effective approach for analysis of droplet impact dynamics associated with piezoelectric drop-ondemand inkjet printing systems was presented. The SPH formulation of Lucy and Gingold and Monaghan has been used as the basis for the model, with the The $\delta$-SPH terms of Marrone et al and gradient correction terms of Belytschko used to improve the accuracy and stability.

Without suitable experimental data to validate against, a series of test cases were used to assess the accuracy of the implemented model, with results showing a good correlation to experimental, analytical and numerical solutions for both macroscale and microscale problems. The model was used to assess the impact dynamics of a single polymer droplet on to a planar surface and subsequently used to assess the formation of a simple single material three dimensional structure through deposition of multiple droplet

Further work is ongoing to better capture cure and non-Newtonian behavior. This detailed model and associated surrogate models will then be used to support a condition based monitoring system to assess and optimize the quality of this additive manufacturing process for electronic components and systems.

\section{Acknowledgements}

This paper is based on work supported by the NextFactory research project funded under the European Community's 7th Framework Programme (FP7/20072013) under grant agreement No. 608985

\section{References}

1. Kawahara ,Y., Hodges, S., Cook, B. S., Zhang, C., Abowd, G. D., "Instant inkjet circuits: lab-based inkjet printing to support rapid prototyping of UbiComp devices", Proc UbiComp'13, September 8-12, 2013, Zurich, Switzerland, pp. 363-372

2. Guenthel, J., Holzinger, D., Birch, R., Refle, O., "Process \& Machine Development for the Integration of Conductive Paths Inside a 3D Printed Microsystem", Proc. Direct Digital Manufacturing Conference, Berlin, Germany, 2016

3. Tourloukis, G., Stoyanov, S., Tilford, T., Bailey, C., "Data driven approach to quality assessment of 3D printed electronic products", Proc. 38th International Spring Seminar on Electronics Technology, 2015, pp. $300-305$

4. Patankar, Suhas V., Numerical Heat Transfer and Fluid Flow, McGraw-Hill, 1980
5. Lucy, L., "A numerical approach to testing the fission hypothesis", Journal Astronomical., Vol. 82, pp. 1013-1924, 1977

6. Gingold, R. A., Monaghan, J. J., "Smoothed Particle Hydrodynamics: Theory and Application to nonSpherical Stars" Mon. Not. R. Astr. Soc., Vol. 181, pp. 375-389, 1977

7. Marrone, S., Antuono, M., Colagrossi, A., Le Touzé, D., Graziani, G., " $\delta$-SPH Model for Simulating Violent Impact Flows", Computer Methods in Applied Mechanics and Engineering. Vol. 200, pp. 1526-1542, 2011

8. Monaghan, J. J., "On the Problem of Penetration in Particle Methods". Journal Computational Physics, Vol. 82, pp. 1-15, 1989

9. Belytschko, T., Krongauz, Y., Organ, D., Fleming, M., "Meshless methods: an overview and recent developments", Computer Methods in Applied Mechanics and Engineering, Vol. 139, Issues 1-4, pp. 3-47, 1996

10. L., Verlet, "Computer Experiments on Classical Fluids, I: Thermodynamical Properties of LennardJones Molecules. Phys Rev, Vol. 159, pp. 98-103, 1967

11. Buchner, B., Green Water on Ship-type Offshore Structures, Ph.D. Thesis, Delft University of Technology, 2002.

12. Young, T., "An essay on the cohesion of fluids," Philosophical Transactions of the Royal Society of London, Vol. 95, pp. 65-87. 1805

13. Laplace, P. S., "Sur l'action capillaire", Traité de Mécanique Céleste, Vol. 4, pp. 1-79., 1805

14. Gauss, C. F., Principia Generalia Theoriae Figurae Fluidorum in Statu Aequilibrii, Dieterichs, 1830 